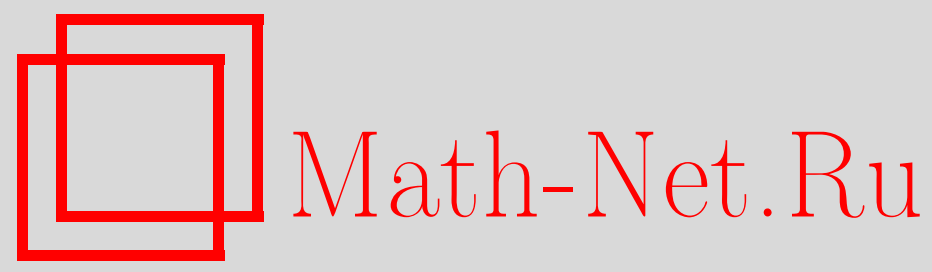

А. С. Терсенов, Задача Дирихле для одного класса квазилинейных эллиптических уравнений, Матем. заметки, 2004, том 76, выпуск 4, 592-603

DOI: https://doi.org/10.4213/mzm134

Использование Общероссийского математического портала Math-Net.Ru подразумевает, что вы прочитали и согласны с пользовательским соглашением http://www . mathnet.ru/rus/agreement

Параметры загрузки:

IP : 52.205 .19 .152

26 апреля 2023 г., 05:46:40

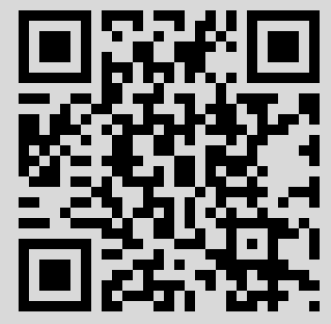




\title{
ЗАДАЧА ДИРИХЛЕ ДЛЯ ОДНОГО КЛАССА КВАЗИЛИНЕЙНЫХ ЭЛЛИПТИЧЕСКИХ УРАВНЕНИЙ
}

\author{
Ал. С. Терсенов
}

В статье исследуется задача Дирихле для квазилинейных эллиптических уравнений. Получены новые априорные оценки решения и градиента решения. Оценки эти получаются без каких-либо предположений о гладкости коэффициентов и правой части уравнения. Кроме того, допускается произвольный рост правой части по градиенту решения. На основе полученных оценок доказываются теоремы существования.

Библиография: 14 названий.

Введение. Данная статья посвящена исследованию задачи Дирихле для квазилинейных эллиптических уравнений. Как известно (см. [1], [2]), доказательство разрешимости краевых задач и, в частности, задачи Дирихле сводится к получению априорной оценки решения $u(\mathbf{x}), \mathbf{x}=\left(x_{1}, \ldots, x_{n}\right)$, в норме пространства $C^{1, \alpha}, \alpha \in(0,1)$ (мы следуем обозначениям, принятым в [2]). Эта процедура разделяется на четыре этапа: оценка $\max |u(\mathbf{x})|$, оценка $\max |\nabla u(\mathbf{x})|$ на границе области, глобальная (т.е. во всей области) оценка $\max |\nabla u(\mathbf{x})|$ и, наконец, оценка $|\nabla u(\mathbf{x})|$ в норме пространства $C^{\alpha}, \nabla u=$ $\left(u_{x_{1}}, \ldots, u_{x_{n}}\right)$. Ключевой здесь является оценка $\max |\nabla u(\mathbf{x})|$, поскольку после ее получения разрешимость краевых задач следует без дополнительных ограничений на характер нелинейности уравнения. Как известно, при получении оценки для $\max |\nabla u(\mathbf{x})|$ в общем случае применяется следующий классический подход (см. [1]-[3]): граничная оценка $\max |\nabla u(\mathbf{x})|$ получается при помощи построения барьеров для $u(\mathbf{x})$ вблизи границы, а глобальная - при помощи дифференцирования и применения принципа максимума к функции $v(\mathbf{x})=|\nabla u(\mathbf{x})|^{2}$. Этот метод восходит к Бернштейну (см. [4]). Очевидно, при таком подходе необходимо требовать дифференцируемость коэффициентов и правой части уравнения, а также накладывать ряд обременительных ограничений на поведение первых производных коэффициентов и правой части (см. [1], [2]). При получении как граничной, так и глобальной оценки градиента, вообще говоря, необходимо требовать вьполнения условия Бернштейна [4] (см. [1]-[3]). Это условие заключается в том, что скорость роста правой части уравнения по $|\nabla u(\mathbf{x})|$ при $|\nabla u(\mathbf{x})| \rightarrow+\infty$ не должна превышать скорости роста главной части по $|\nabla u(\mathbf{x})|$ более, чем на $|\nabla u(\mathbf{x})|^{2}$. Известно (см., например, [5]), что для уравнения $\Delta u=f(\mathbf{x}, u, \nabla u)$ в случае непрерьвной функции $f(\mathbf{x}, u, \mathbf{p})$ вьполнения условия Бернштейна достаточно для того, чтобы из оценки $\max |u|$ вытекала оценка $\max |\nabla u|$. Похожаев в [6] показал, что если вместо непрерьвности функции $f$ потребовать вьполнение более слабого условия: $f \in L_{q}(\Omega)$, $q>n$, при $u \in W_{q}^{2}(\Omega)$, то условие Бернштейна уже не будет достаточньм для получения оценки $\max |\nabla u|$ из $\max |u|$. В [6] сформулировано условие на рост функции $f(\mathbf{x}, u, \mathbf{p})$ по 
$\mathbf{p}$, при которых оценка $\max |u|$ влечет оценку $\max |\nabla u|$. Это условие зависит от $q$ и переходит в условие Бернштейна при $q=+\infty$. Что касается оценки $\max |u(\mathbf{x})|$, то существует ряд достаточных условий, гарантирующих такую оценку, см. [1], [2], [7]. Отметим, что в [7] рассматриваются параболические уравнения, однако результаты, полученные там, легко переносятся и на эллиптический случай.

Настоящая статья посвящена получению граничной и глобальной априорной оценки для $\max |\nabla u(\mathbf{x})|$ в случае, когда условие Бернштейна не вьполнено. При получении глобальной оценки используется метод введения дополнительной переменной, предложенньй Кружковым (см. [8]) для исследования квазилинейных параболических уравнений с одной пространственной переменной (об этом методе см. также [9]-[14]). На основе метода Кружкова удается получить глобальную априорную оценку для $|\nabla u(\mathbf{x})|$ без дифференцирования для одного класса квазилинейных эллиптических уравнений со многими независимыми переменными. Специфика этого класса уравнений заключается, в частности, в том, что коэффициенты не зависят явно от самого решения и от части пространственных переменных. Предполагаем, что область, в которой решается задача Дирихле, является вьпуклой. Основное отличие от аналогичной оценки в [12] заключается в том, что допускается произвольньй рост правой части по $|\nabla u(\mathbf{x})|$ независимо от роста главной части уравнения. Приведем в качестве примера следующую задачу:

$$
\Delta u=f_{1}(\mathbf{x})+\phi_{1}(u) \phi_{2}(\nabla u) \text { в } \Omega, \quad u=0 \text { на } \partial \Omega .
$$

Здесь $f_{1}(\mathbf{x})$ - ограниченная в $\bar{\Omega}$ функция, $\phi_{1}(u)$ - неубывающая функция, $u \phi_{1}(u) \geqslant 0$ и $\phi_{2}(\nabla u) \geqslant 0$. Например, $\phi_{1}=u^{3}$ либо $\phi_{1}=u|u|^{1 / 3}$, a $\phi_{2}=e^{|\nabla u|} \sqrt{|\nabla u|}+1$ либо $\phi_{2}=\left|u_{x_{1}}\right|^{k_{1}}+\cdots+\left|u_{x_{n}}\right|^{k_{n}}+k_{0}$, где $k_{i}$ - неотрицательные вешественные числа.

Как было показано Ладыженской и Уральцевой (см. [1]), после получения оценок для $\max |u(\mathbf{x})|$ и $\max |\nabla u(\mathbf{x})|$ оценка $|\nabla u(\mathbf{x})|$ в норме пространства $C^{\alpha}$ получается при определенных условиях на гладкость коэффициентов и правой части уравнения.

В п. 1 для простоты изложения все рассуждения ведутся для уравнения $\Delta u=f(\mathbf{x}, u$, $\nabla u)$. Сформулированы теоремы существования решения, которые вытекают из полученных оценок. В частности, существование классического решения задачи (1) гарантируется, если $f_{1} \in C^{\alpha}(\bar{\Omega}), \phi_{1} \in C^{\alpha}(\mathbb{R}), \phi_{2} \in C^{\alpha}\left(\mathbb{R}^{n}\right)$ для некоторого $\alpha \in(0,1)$.

Уравнениям с двумя независимыми переменными посвящен п. 2. Здесь рассмотрено общее двумерное уравнение и, кроме того, приводится многомерное квазилинейное уравнение, на которое распространяются результаты второго пункта.

1. Многомерный случай. Для простоты все рассуждения будем вести при $n=3$, случай $n>3$ может быть исследован аналогично. Пусть $\Omega \subset \mathbb{R}^{3}-$ строго вьпуклая область и $\partial \Omega \in C^{2+\alpha}$, где $\alpha \in(0,1)$. Не ограничивая общности, считаем, что, во-первых, точка $(0,0,0) \in \Omega$, во-вторых, части $\partial \Omega$, лежащие в полупространствах $x_{1} \leqslant 0, x_{1} \geqslant 0$, можно представить в виде

$$
x_{1}=F_{1}\left(x_{2}, x_{3}\right), \quad x_{1}=G_{1}\left(x_{2}, x_{3}\right),
$$

а части $\partial \Omega$, лежащие в полупространствах $x_{2} \leqslant 0, x_{2} \geqslant 0$ и $x_{3} \leqslant 0, x_{3} \geqslant 0$, можно представить в виде

$$
x_{2}=F_{2}\left(x_{1}, x_{3}\right), \quad x_{2}=G_{2}\left(x_{1}, x_{3}\right) \quad \text { и } \quad x_{3}=F_{3}\left(x_{1}, x_{2}\right), \quad x_{3}=G_{3}\left(x_{1}, x_{2}\right)
$$

соответственно, где $F_{i}, G_{i}$ - дважды непрерьвно дифференцируемые функции и, в-третьих, $x_{i}$ меняется в пределах от $-l_{i}$ до $l_{i}, i=1,2,3$. 
Рассмотрим следующую задачу Дирихле:

$$
\begin{aligned}
\Delta u & =f(\mathbf{x}, u, \nabla u) \quad \text { в } \Omega \subset \mathbb{R}^{3}, \\
u & =0 \quad \text { на } \partial \Omega,
\end{aligned}
$$

где $f$ представима в виде

$$
f(\mathbf{x}, u, \mathbf{p})=f_{1}(\mathbf{x}, u, \mathbf{p})+f_{2}(\mathbf{x}, u, \mathbf{p}) .
$$

Предположим, что при $\mathbf{x} \in \Omega,|u| \leqslant M$ и конечных $\mathbf{p}=\left(p_{1}, p_{2}, p_{3}\right)$ функция $f_{1}$ удовлетворяет структурному ограничению

$$
\left|f_{1}(\mathbf{x}, u, \mathbf{p})\right|<\psi\left(\left|p_{1}\right|\right),
$$

где $\psi(\rho)>0$ - непрерывно дифференцируемая функция такая, что

$$
\int_{0}^{+\infty} \frac{\rho d \rho}{\psi(\rho)}>\mu_{1} \equiv \max \{M, \operatorname{osc}(u)\}
$$

а функция $f_{2}$ удовлетворяет соотношению

$$
u f_{2}(\mathbf{x}, u, \mathbf{p}) \geqslant 0 .
$$

Введем функции $h_{1}\left(x_{1}\right)$ и $h_{2}\left(x_{1}\right) \equiv h_{1}\left(-x_{1}\right)$ :

$$
h_{1}^{\prime \prime}+\psi\left(\left|h_{1}^{\prime}\right|\right)=0, \quad h_{1}\left(-l_{1}\right)=0, \quad h_{1}\left(-l_{1}+\tau_{0}\right)=\mu_{1},
$$

постоянная $\tau_{0}$ будет выбрана ниже. Представим $h_{1}$ в параметрическом виде:

$$
h_{1}=h_{1}(q)=\int_{q}^{q_{1}} \frac{\rho d \rho}{\psi(\rho)}, \quad x_{1}=x_{1}(q)=\int_{q}^{q_{1}} \frac{d \rho}{\psi(\rho)}-l_{1},
$$

где параметр $q$ лежит в интервале $\left[q_{0}, q_{1}\right]$, причем $q_{1}>q_{0}>0$ и

$$
h_{1}\left(q_{0}\right)=\int_{q_{0}}^{q_{1}} \frac{\rho d \rho}{\psi(\rho)}=\mu_{1} .
$$

Это возможно в силу условия (1.5). Положим

$$
\tau_{0}=\int_{q_{0}}^{q_{1}} \frac{d \rho}{\psi(\rho)} .
$$

Очевидно, $h_{1}^{\prime}>0, h_{2}^{\prime}<0$.

Лемма 1.1. Пусть и(х) - классическое решение задачи (1.1), (1.2) такое, что $|\nabla u|<+\infty$ в $\bar{\Omega}$. Предположим, что условия (1.3), (1.4), (1.6) выполнены. Тогда

$$
|u(\mathbf{x})| \leqslant h_{k}\left(\zeta_{k}\right) \quad \forall \mathbf{x} \in \bar{D}_{k}, \quad k=1,2,
$$

əде $\zeta_{1}=x_{1}-F_{1}-l_{1}, \zeta_{2}=x_{1}-G_{1}+l_{1} u$

$$
\begin{aligned}
& D_{1}=\left\{\mathbf{x}: F_{1}\left(x_{2}, x_{3}\right)<x_{1}<F_{1}\left(x_{2}, x_{3}\right)+\tau_{0},\left(x_{2}, x_{3}\right) \in \Omega_{1}\right\} \cap \Omega, \\
& D_{2}=\left\{\mathbf{x}: G_{1}\left(x_{2}, x_{3}\right)-\tau_{0}<x_{1}<G_{1}\left(x_{2}, x_{3}\right),\left(x_{2}, x_{3}\right) \in \Omega_{1}\right\} \cap \Omega,
\end{aligned}
$$

$\Omega_{1}-$ проекиия области $\Omega$ на плоскость $\left(x_{2}, x_{3}\right)$. 
ДоКАЗАТЕЛЬСТВо. Если $\tau_{0} \geqslant 2 l_{1}$, то $\partial D_{1}=\partial D_{2}=\partial \Omega$ и в силуграничного условия $\left.u\right|_{\partial \Omega}=0 \leqslant h_{k}, k=1,2$.

Если $\tau_{0}<2 l_{1}$, то $\partial D_{1}$ состоит из двух частей: $\Gamma_{1}$ и $\Gamma_{2}$, где $\Gamma_{1} \subset \partial \Omega$, а $\Gamma_{2}-$ та часть поверхности $x_{1}=F_{1}\left(x_{2}, x_{3}\right)+\tau_{0}$, которая лежит в $\Omega$. На $\Gamma_{1}$ имеем $u=0 \leqslant h_{1}$, а на $\Gamma_{2}$ получаем $h_{1}\left(-l_{1}+\tau_{0}\right)=\mu_{1} \geqslant u$. Аналогично, при $\tau_{0} \leqslant 2 l_{1}$ поверхность $\partial D_{2}=\Gamma_{3} \cup \Gamma_{4}$, где $\Gamma_{3} \subset \partial \Omega$, а $\Gamma_{4}$ - та часть поверхности $x_{1}=G_{1}\left(x_{2}, x_{3}\right)-\tau_{0}$, которая лежит в $\Omega$. На $\Gamma_{3}$ имеем $u=0 \leqslant h_{2}$, а на $\Gamma_{4}$ получаем $h_{2}\left(l_{1}-\tau_{0}\right)=\mu_{1} \geqslant u$. Следовательно, $|u(\mathbf{x})| \leqslant h_{k}\left(\zeta_{k}\right)$ на $\partial D_{k}$.

В $D_{k}$ имеем

$$
\Delta h_{k}\left(\zeta_{k}\right)=h_{k}^{\prime \prime}+h_{k x_{2} x_{2}}+h_{k x_{3} x_{3}} \leqslant-\psi\left(\left|h_{k}^{\prime}\right|\right), \quad k=1,2
$$

поскольку для $i=2,3$ имеем

$$
\begin{aligned}
& h_{1 x_{i} x_{i}}\left(\zeta_{1}\right)=-\left(F_{1 x_{i}}^{2} \psi\left(\left|h_{1}^{\prime}\left(x_{1}-F_{1}-l_{1}\right)\right|\right)+F_{1 x_{i} x_{i}} h_{1}^{\prime}\left(x_{1}-F_{1}-l_{1}\right)\right) \leqslant 0, \\
& h_{2 x_{i} x_{i}}\left(\zeta_{2}\right)=-\left(G_{1 x_{i}}^{2} \psi\left(\left|h_{2}^{\prime}\left(x_{1}-G_{1}+l_{1}\right)\right|\right)+G_{1 x_{i} x_{i}} h_{2}^{\prime}\left(x_{1}-G_{1}+l_{1}\right)\right) \leqslant 0 .
\end{aligned}
$$

В последнем нетрудно убедиться, если вспомнить, что $h_{1}^{\prime} \geqslant 0$, a $h_{2}^{\prime} \leqslant 0$ и, кроме того, в силу вьпуклости области, $F_{1 x_{i} x_{i}} \geqslant 0, G_{1 x_{i} x_{i}} \leqslant 0$. Следовательно, для $v \equiv u-h_{k}$ получаем

$$
\Delta v \geqslant f(\mathbf{x}, u, \nabla u)+\psi\left(\left|h_{k}^{\prime}\right|\right) .
$$

Если функция $v$ достигает своего наибольшего значения в точке $N \in \bar{D}_{k} \backslash \partial D_{k}$, тогда в этой точке $v>0, v_{x_{i}}=0$, т.е. $u>h_{k} \geqslant 0, \nabla u=\nabla h_{k}$, следовательно, из (1.4), (1.6) и (1.8) получаем

$$
\left.\Delta v\right|_{N} \geqslant f_{1}(\mathbf{x}, u, \nabla u)+\psi\left(\left|u_{x_{1}}\right|\right)+\left.f_{2}(\mathbf{x}, u, \nabla u)\right|_{N}>0 .
$$

Это противоречит предположению о достижении функцией $v$ максимума во внутренних точках области $D_{k}$. Следовательно,

$$
u(\mathbf{x}) \leqslant h_{k}\left(\zeta_{k}\right) \text { в } \bar{D}_{k}, \quad k=1,2 .
$$

Теперь получим оценку снизу. Для $w \equiv u+h_{k}$ имеем

$$
\left.\Delta w \leqslant f(\mathbf{x}, u, \nabla u)-\psi\left(\mid h_{k}^{\prime}\right) \mid\right) .
$$

Если функция $w$ принимает наименьшее значение в точке $N_{1} \in \bar{D}_{k} \backslash \partial D_{k}$, то в этой точке $u<0, \nabla u=-\nabla h_{k}$ и, следовательно, в силу (1.4) и (1.6) из (1.9) получаем

$$
\left.\Delta w\right|_{N_{1}} \leqslant f_{1}(\mathbf{x}, u, \nabla u)-\psi\left(\left|u_{x_{1}}\right|\right)+\left.f_{2}(\mathbf{x}, u, \nabla u)\right|_{N_{1}}<0 .
$$

Это противоречит предположению, что $w$ принимает минимум во внутренних точках области $D_{k}$. Следовательно, $u(\mathbf{x}) \geqslant-h_{k}\left(\zeta_{k}\right)$ в $\bar{D}_{k}, k=1,2$. Лемма доказана.

Приступим теперь к получению глобальной оценки $\left|u_{x_{1}}\right|$. Предположим, что $f_{2}$ дополнительно удовлетворяет соотношениям

$$
\begin{array}{r}
f_{2}\left(x_{1}, x_{2}, x_{3}, u, p_{1}, p_{2}, p_{3}\right)-f_{2}\left(\zeta, x_{2}, x_{3}, v, p_{1}, p_{2}, p_{3}\right) \geqslant 0 \\
f_{2}\left(\zeta, x_{2}, x_{3}, u,-p_{1}, p_{2}, p_{3}\right)-f_{2}\left(x_{1}, x_{2}, x_{3}, v,-p_{1}, p_{2}, p_{3}\right) \geqslant 0
\end{array}
$$

при $x_{1}>\zeta, u>v, p_{1}>0$ и любых $p_{2}, p_{3}$. 
ЛЕмма 1.2. Пусть выполнены предположения леммы 1.1 и условия (1.10). Тогда для $и(\mathbf{x})$ имеет место оценка

$$
\sup _{\bar{\Omega}}\left|u_{x_{1}}\right| \leqslant C_{1}
$$

где постоянная $C_{1}$ зависит лишь от $\psi$ и $M$.

ДокАЗАтЕльство. Рассмотрим уравнение (1.1) в двух различных точках области $\Omega: \mathbf{x}=\left(x_{1}, x_{2}, x_{3}\right)$ и $\left(\zeta, x_{2}, x_{3}\right)$. Имеем

$$
\begin{array}{rll}
u_{x_{1} x_{1}}+u_{x_{2} x_{2}}+u_{x_{3} x_{3}} & =f^{\left(x_{1}\right)} \quad \text { для } \quad u=u(\mathbf{x}), \\
u_{\zeta \zeta}+u_{x_{2} x_{2}}+u_{x_{3} x_{3}}=f^{(\zeta)} & \text { для } \quad u=u\left(\zeta, x_{2}, x_{3}\right),
\end{array}
$$

где $f^{(z)} \equiv f\left(z, x_{2}, x_{3}, u\left(z, x_{2}, x_{3}\right), \nabla u\left(z, x_{2}, x_{3}\right)\right)$. Вычитая уравнение (1.12) из уравнения (1.11), для функции $v(\mathrm{x}, \zeta) \equiv u\left(x_{1}, x_{2}, x_{3}\right)-u\left(\zeta, x_{2}, x_{3}\right)$ получаем

$$
\Delta_{\mathbf{x}, \zeta} v \equiv v_{x_{1} x_{1}}+v_{\zeta \zeta}+v_{x_{2} x_{2}}+v_{x_{3} x_{3}}=f^{\left(x_{1}\right)}-f^{(\zeta)} .
$$

Пусть функция $h(\tau) \equiv h\left(x_{1}-\zeta\right)$ является решением задачи

$$
h^{\prime \prime}+\psi\left(\left|h^{\prime}\right|\right)=0, \quad h(0)=0, \quad h\left(\tau_{0}\right)=\mu_{1},
$$

величина $\tau_{0}$ была определена при построении барьера $h_{1}($ см. (1.7)). Очевидно,

$$
\Delta_{\mathbf{x}, \zeta} h\left(x_{1}-\zeta\right)=2 h^{\prime \prime}=-2 \psi\left(\left|h^{\prime}\right|\right)
$$

Для функции $w \equiv v-h\left(x_{1}-\zeta\right)$ получаем

$$
\begin{aligned}
\Delta_{\mathbf{x}, \zeta} w= & f^{\left(x_{1}\right)}-f^{(\zeta)}-2 \psi\left(\left|h^{\prime}\right|\right) \\
= & f_{1}(\mathbf{x}, u(\mathbf{x}), \nabla u(\mathbf{x}))-\psi\left(\left|h^{\prime}\right|\right) \\
& -\left(f_{1}\left(\zeta, x_{2}, x_{3}, u\left(\zeta, x_{2}, x_{3}\right), \nabla u\left(\zeta, x_{2}, x_{3}\right)\right)-\psi\left(\left|h^{\prime}\right|\right)\right) \\
& +f_{2}(\mathbf{x}, u(\mathbf{x}), \nabla u(\mathbf{x}))-f_{2}\left(\zeta, x_{2}, x_{3}, u\left(\zeta, x_{2}, x_{3}\right), \nabla u\left(\zeta, x_{2}, x_{3}\right)\right) .
\end{aligned}
$$

Рассмотрим область $Q$ :

$$
Q=\left\{(\mathbf{x}, \zeta): x_{1} \in\left(F_{1}, G_{1}\right), \zeta \in\left(F_{1}, G_{1}\right), 0<x_{1}-\zeta<\tau_{0},\left(x_{2}, x_{3}\right) \in \Omega_{1}\right\}
$$

(напомним, что $\Omega_{1}$ есть проекция $\Omega$ на плоскость $\left(x_{2}, x_{3}\right)$ ). Если $\tau_{0} \geqslant 2 l_{1}$, то полагаем

$$
Q=\left\{(\mathbf{x}, \zeta): x_{1} \in\left(F_{1}, G_{1}\right), \zeta \in\left(F_{1}, G_{1}\right), 0<x_{1}-\zeta,\left(x_{2}, x_{3}\right) \in \Omega_{1}\right\}
$$

Предположим, что функция $w$ принимает наибольшее значение во внутренней точке $N$ области $Q(N \in \bar{Q} \backslash \partial Q)$. Очевидно, в $N$ функция $w>0, \nabla w=0$ и, следовательно, $u \geqslant 0, u_{x_{1}}(\mathbf{x})=h^{\prime}\left(x_{1}-\zeta\right), u_{\zeta}\left(\zeta, x_{2}, x_{3}\right)=h^{\prime}\left(x_{1}-\zeta\right), u_{x_{i}}(\mathbf{x})=u_{x_{i}}\left(\zeta, x_{2}, x_{3}\right), i=2,3$, т.е.

$$
\left.\nabla u(\mathbf{x})\right|_{N}=\left.\nabla u\left(\zeta, x_{2}, x_{3}\right)\right|_{N} .
$$

Таким образом, из $(1.4),\left(1.10_{1}\right)$ и $(1.13)$ получаем $\left.\Delta_{\mathbf{x}, \zeta} w\right|_{N}>0$, что противоречит предположению о том, что $w$ принимает максимум во внутренней точке области $Q$. 
Покажем, что $w \leqslant 0$ на $\partial Q$.

1) Для $x_{1}=\zeta$ имеем $v=h=0$.

2) При $x_{1}-\zeta=\tau_{0}$ получаем $h\left(\tau_{0}\right)=\mu_{1} \geqslant u(\mathbf{x})-u\left(\zeta, x_{2}, x_{3}\right)$. Эта часть гранищы присутствует лишь в случае $\tau_{0}<2 l_{1}$.

3) При $\zeta=F_{1}\left(x_{2}, x_{3}\right), x_{1} \in\left[F_{1}, F_{1}+\tau_{0}\right] \cap\left[F_{1}, G_{1}\right],\left(x_{2}, x_{3}\right) \in \Omega_{1}$ выполняется $v-h=u(\mathbf{x})-h\left(x_{1}-F_{1}\right)$. Покажем теперь, что $u(\mathbf{x}) \leqslant h\left(x_{1}-F_{1}\right)$. Для этого достаточно получить равенство $h\left(x_{1}-F_{1}\right)=h_{1}\left(x_{1}-F_{1}-l_{1}\right)$, а затем применить лемму 1.1. Требуемое равенство непосредственно следует из того, что

$$
\begin{gathered}
h_{1}^{\prime \prime}\left(\zeta_{1}\right)+\psi\left(\left|h_{1}^{\prime}\left(\zeta_{1}\right)\right|\right)=0, \quad h_{1}\left(-l_{1}\right)=0, \quad h_{1}\left(-l_{1}+\tau_{0}\right)=\mu_{1}, \quad \zeta_{1}=x_{1}-F_{1}-l_{1}, \\
h^{\prime \prime}(\eta)+\psi\left(\left|h^{\prime}(\eta)\right|\right)=0, \quad h(0)=0, \quad h\left(\tau_{0}\right)=\mu_{1}, \quad \eta=x_{1}-F_{1} .
\end{gathered}
$$

4) При $x_{1}=G_{1}\left(x_{2}, x_{3}\right), \zeta \in\left[G_{1}-\tau_{0}, G_{1}\right] \cap\left[F_{1}, G_{1}\right],\left(x_{2}, x_{3}\right) \in \Omega_{0}$ получаем

$$
v-h=-u\left(\zeta, x_{2}, x_{3}\right)-h\left(G_{1}-\zeta\right)
$$

Покажем, что $u(\zeta, y) \geqslant-h\left(G_{1}(y)-\zeta\right)$. Достаточно убедиться в том, что $h\left(G_{1}-\zeta\right)=$ $h_{2}\left(\zeta-G_{1}+l_{1}\right)$, а затем вновь применить лемму 1.1. Последнее равенство непосредственно вытекает из того, что

$$
\begin{gathered}
h_{2}^{\prime \prime}\left(\zeta_{2}\right)+\psi\left(\left|h_{2}^{\prime}\left(\zeta_{2}\right)\right|\right)=0, \quad h_{2}\left(l_{1}-\tau_{0}\right)=\mu_{1}, \quad h_{2}\left(l_{1}\right)=0, \quad \zeta_{2}=\zeta-G_{1}+l_{1}, \\
h^{\prime \prime}(\tilde{\eta})+\psi\left(\left|h^{\prime}(\tilde{\eta})\right|\right)=0, \quad h(0)=0, \quad h\left(\tau_{0}\right)=\mu_{1}, \quad \tilde{\eta}=G_{1}-\zeta .
\end{gathered}
$$

Таким образом, $w \leqslant 0$ на $\partial Q$. Благодаря тому, что $w$ не может принимать максимум в $\bar{Q} \backslash \partial Q$, получаем, что $u(\mathbf{x})-u\left(\zeta, x_{2}, x_{3}\right) \leqslant h\left(x_{1}-\zeta\right)$ в $\bar{Q}$.

Аналогично, взяв функцию $\tilde{v} \equiv u\left(\zeta, x_{2}, x_{3}\right)-u(\mathbf{x})$ вместо $v$, получаем $v \geqslant-h\left(x_{1}-\zeta\right)$ в $\bar{Q}$ (здесь мы используем условие $\left.\left(1.10_{2}\right)\right)$.

В силу симметрии переменных $x_{1}$ и $\zeta$ точно так же рассматриваем случай $\zeta>x_{1}$. В результате имеем, что для $x_{1} \in\left[F_{1}, G_{1}\right], \zeta \in\left[F_{1}, G_{1}\right],\left(x_{2}, x_{3}\right) \in \Omega_{1}, 0<\left|x_{1}-\zeta\right|<\tau_{0}$ выполняется неравенство

$$
\frac{\left|u(\mathbf{x})-u\left(\zeta, x_{2}, x_{3}\right)\right|}{\left|x_{1}-\zeta\right|} \leqslant \frac{h\left(\left|x_{1}-\zeta\right|\right)-h(0)}{\left|x_{1}-\zeta\right|}
$$

которое, в свою очередь, влечет оценку $\left|u_{x_{1}}(\mathbf{x})\right| \leqslant h^{\prime}(0)=C_{1}$. Лемма доказана.

Сформулируем теперь условия, обеспечивающие априорную оценку $u_{x_{2}}$.

Предположим, что функция $f_{1}$ при $\mathbf{x} \in \Omega,|u| \leqslant M,\left|p_{1}\right| \leqslant C_{1}$ и конечных $p_{2}, p_{3}$ удовлетворяет неравенству

$$
\left|f_{1}(\mathbf{x}, u, \mathbf{p})\right|<\psi\left(\left|p_{2}\right|\right) .
$$

Введем функции $h_{3}\left(x_{2}\right), h_{4}\left(x_{2}\right)$ следуюшим образом: $h_{4}\left(x_{2}\right)=h_{3}\left(-x_{2}\right)$,

$$
h_{3}^{\prime \prime}+\psi\left(\left|h_{3}^{\prime}\right|\right)=0, \quad h_{3}\left(-l_{2}\right)=0, \quad h_{3}\left(-l_{2}+\tau_{0}\right)=\mu_{1} .
$$

Доказательство следующей леммы аналогично доказательству леммы 1.1. 
ЛЕмма 1.3. Пусть выполнены условия лемм 1.1, 1.2 и условие (1.14). Тогда

$$
|u(\mathbf{x})| \leqslant h_{k}\left(\zeta_{k}\right) \quad \forall \mathbf{x} \in \bar{D}_{k}, \quad k=3,4,
$$

әде $\zeta_{3}=x_{2}-F_{2}-l_{2}, \zeta_{4}=x_{2}-G_{2}+l_{2}$,

$$
\begin{aligned}
& D_{3}=\left\{\mathbf{x}: F_{2}<x_{2}<F_{2}+\tau_{0},\left(x_{1}, x_{3}\right) \in \Omega_{2}\right\} \cap \Omega, \\
& D_{4}=\left\{\mathbf{x}: G_{2}-\tau_{0}<x_{2}<G_{2},\left(x_{1}, x_{3}\right) \in \Omega_{2}\right\} \cap \Omega,
\end{aligned}
$$

$a \Omega_{2}$ - проекция $\Omega$ на плоскость $\left(x_{1}, x_{3}\right)$.

Предположим дополнительно, что

$$
\begin{array}{r}
f_{2}\left(x_{1}, x_{2}, x_{3}, u, p_{1}, p_{2}, p_{3}\right)-f_{2}\left(x_{1}, \zeta, x_{3}, v, p_{1}, p_{2}, p_{3}\right) \geqslant 0 \\
f_{2}\left(x_{1}, \zeta, x_{3}, u, p_{1},-p_{2}, p_{3}\right)-f_{2}\left(x_{1}, x_{2}, x_{3}, v, p_{1},-p_{2}, p_{3}\right) \geqslant 0
\end{array}
$$

при $x_{2}>\zeta, u>v, p_{2}>0$ и любых $p_{1}, p_{3}$.

ЛЕмма 1.4. Пусть выполнены условия (1.15) и условия лемм 1.1-1.3. Тогда для $u(\mathbf{x})$ выполняется оценка

$$
\sup _{\bar{\Omega}}\left|u_{x_{2}}\right| \leqslant C_{2}
$$

где постоянная $C_{2}$ зависит лишь от $\psi$ и $M$.

Доказательство этой леммы аналогично доказательству леммы 1.2 .

Перейдем к оценке $u_{x_{3}}$. Предположим, что функция $f_{1}$ при $\mathbf{x} \in \Omega,|u| \leqslant M,\left|p_{1}\right| \leqslant C_{1}$, $\left|p_{2}\right| \leqslant C_{2}$ и конечных $p_{3}$ удовлетворяет условию

$$
\left|f_{1}(\mathbf{x}, u, \mathbf{p})\right|<\psi\left(\left|p_{3}\right|\right) \text {. }
$$

Введем функции $h_{5}\left(x_{3}\right), h_{6}\left(x_{3}\right)$ следующим образом: $h_{6}\left(x_{3}\right)=h_{5}\left(-x_{3}\right)$

$$
h_{5}^{\prime \prime}+\psi\left(\left|h_{5}^{\prime}\right|\right)=0, \quad h_{5}\left(-l_{3}\right)=0, \quad h_{5}\left(-l_{3}+\tau_{0}\right)=\mu_{1} .
$$

Доказательство следующей леммы аналогично доказательству леммы 1.1.

ЛЕмма 1.5. Пусть выполнены условия лемм 1.1-1.4 и условие (1.16). Тогда

$$
|u(\mathbf{x})| \leqslant h_{k}\left(\zeta_{k}\right) \quad \forall \mathbf{x} \in \bar{D}_{k}, \quad k=5,6,
$$

əде $\zeta_{5}=x_{3}-F_{3}-l_{3}, \zeta_{6}=x_{3}-G_{3}+l_{3}$,

$$
\begin{aligned}
& D_{5}=\left\{\mathbf{x}: F_{3}<x_{3}<F_{3}+\tau_{0},\left(x_{1}, x_{2}\right) \in \Omega_{3}\right\} \cap \Omega, \\
& D_{6}=\left\{\mathbf{x}: G_{3}-\tau_{0}<x_{3}<G_{3},\left(x_{1}, x_{2}\right) \in \Omega_{3}\right\} \cap \Omega,
\end{aligned}
$$

$a \Omega_{3}$ - проекция $\Omega$ на плоскость $\left(x_{1}, x_{2}\right)$.

Для получения глобальной оценки величины $u_{x_{3}}$ предположим, что

$$
\begin{array}{r}
f_{2}\left(x_{1}, x_{2}, x_{3}, u, p_{1}, p_{2}, p_{3}\right)-f_{2}\left(x_{1}, x_{2}, \zeta, v, p_{1}, p_{2}, p_{3}\right) \geqslant 0 \\
f_{2}\left(x_{1}, x_{2}, \zeta, u, p_{1}, p_{2},-p_{3}\right)-f_{2}\left(x_{1}, x_{2}, x_{3}, v, p_{1}, p_{2},-p_{3}\right) \geqslant 0
\end{array}
$$

при $x_{3}>\zeta, u>v, p_{3}>0$ и любых $p_{1}, p_{2}$. 
ЛЕмма 1.6. Пусть выполнены условия (1.17) и условия лемм 1.1-1.5. Тогда для $u(\mathbf{x})$ выполняется

$$
\sup _{\bar{\Omega}}\left|u_{x_{3}}\right| \leqslant C_{3}
$$

где постоянная $C_{3}$ зависит лищь от $\psi$ и $M$.

Доказательство этой леммы аналогично доказательству леммы 1.2.

ЗАмЕчАнИЕ 1.1 . В леммах $1.2,1.4$ и 1.6 получены оценки $\left|u_{x_{i}}\right|$, зависящие лишш от $M=\max |u|$ и $\psi$, в предположении, что $\psi$ удовлетворяет условию (1.5). Нетрудно показать, что если условие (1.5) заменить следующим:

$$
\int_{0}^{+\infty} \frac{d \rho}{\psi(\rho)}>2 l_{i}
$$

то можно получить оценки $\left|u_{x_{i}}\right|$, зависящие лишь от $\psi$ и $l_{i}$. Для этого необходимо внести некоторые изменения в построение барьеров $h_{i}\left(x_{i}\right)$ и $h(\tau)$. А именно $h_{i}$ и $h$ должны быть решениями задач

$$
\begin{gathered}
h_{i}^{\prime \prime}+\psi\left(\left|h_{i}^{\prime}\right|\right)=0, \quad h_{i}\left(-l_{i}\right)=0, \quad h_{i}\left(l_{i}\right)=H, \quad i=1,3,5, \\
h^{\prime \prime}+\psi\left(\left|h^{\prime}\right|\right)=0, \quad h(0)=0, \quad h\left(2 l_{i}\right)=H
\end{gathered}
$$

соответственно. Представим решение задачи (1.19) в параметрическом виде:

$$
h(q)=\int_{q}^{q_{1}} \frac{\rho d \rho}{\psi(\rho)}, \quad \tau(q)=\int_{q}^{q_{1}} \frac{d \rho}{\psi(\rho)} .
$$

Параметр $q \in\left[q_{0}, q_{1}\right]$, а $q_{0}, q_{1}$ выбраны так, что $0 \leqslant q_{0}<q_{1}<+\infty$ и $\tau\left(q_{0}\right)=2 l_{1}$, что возможно в силу (1.18). Оценка

$$
|u(\mathbf{x})| \leqslant h_{k}\left(\zeta_{k}\right), \quad k=1, \ldots, 6
$$

получается аналогично лемме 1.1 ; отметим, что в данном случае $\tau_{0} \equiv \tau\left(q_{0}\right)=2 l_{i}$ и $D_{i}=\Omega, i=1, \ldots, 6$. Глобальная оценка получается аналогично лемме 1.2.

Сформулируем теорему существования и единственности.

Теорема 1.1. Предположими, что $f \in C^{\alpha}\left(\Omega \times \mathbb{R} \times \mathbb{R}^{3}\right)$ для некоторого $\alpha \in(0,1)$ и выполнены условия (1.3), (1.4), (1.6), (1.10), (1.14)-(1.17). Предположим также, что выполнено условие, обеспечивающее априорную оченку $|u| \leqslant M$. Тогда в любой строго выпуклой области $\Omega$ такой, что $\partial \Omega \in C^{2, \alpha}$, задача (1.1), (1.2) имеет по крайней мере одно решение $u(\mathbf{x}) \in C^{2, \alpha}(\bar{\Omega})$. Если функция $f$ возрастает по переменной $и$, то такое решение единственно.

ДокАЗАТЕЛЬСТво. Сушествование такого решения следует из полученных вьше априорных оценок и теоремы 13.8 из [2]. В [2, теорема 10.2] единственность классического решения доказьвается в предположении независимости функции $a_{i j}$ от $u$, дифференцируемости $a_{i j}$ и $f$ по р и убывания функции $f$ по переменной $u$. От условия дифференцируемости по р можно легко избавиться, действуя так же, как в доказательстве теоремы 2.1 из [13].

Приведем два примера. Сначала остановимся на задаче (1), сформулированной во введении. Оценка $\max |u|$ следует, например, из [7]. Нетрудно видеть, что условия (1.4), 
(1.6), (1.10), (1.14)-(1.17) вьполнены. Таким образом, если $f_{1}(\mathbf{x}) \in C^{\alpha}(\bar{\Omega}), f_{2} \equiv$ $\phi_{1}(u) \phi_{2}(\nabla u) \in C^{\alpha}\left(\mathbb{R}^{4}\right)$, то теорема 1.1 гарантирует существование решения задачи $(1)$, принадлежащее пространству $C^{2, \alpha}(\bar{\Omega})$.

Рассмотрим теперь следующее уравнение:

$$
\Delta u=f_{1}\left(\mathbf{x}, u, u_{x_{1}}\right)+f_{2}(\mathbf{x}, u, \nabla u),
$$

где $\left|f_{1}\left(\mathbf{x}, u, p_{1}\right)\right| \leqslant \widetilde{K}\left(1+p_{1}^{2}\right)$ при $\mathbf{x} \in \Omega,|u| \leqslant M$ и любом $p_{1}$. В качестве $f_{2}$ можно взять такую же функцию, как и в предыдушем примере. Для классического решения задачи $(1.20),(1.2)$ такого, что $|\nabla u|<+\infty$, верны оценки лемм 1.1-1.6. Условие (1.4) выполняется с $\psi\left(p_{1}\right)=K_{1}\left(1+p_{1}^{2}\right)$, где $K_{1}>\widetilde{K}$. Из леммы 1.2 следует оценка $\left|u_{x_{1}}\right| \leqslant C_{1}$. Условия (1.14), (1.16) выполнены с $\psi \equiv K_{2}>\max \{\tilde{K}, F\}$, где $F=\max \left|f_{1}\left(\mathbf{x}, u, p_{1}\right)\right|$, максимум функции $\left|f_{1}\left(\mathbf{x}, u, p_{1}\right)\right|$ берется по множеству $\bar{\Omega} \times[-M, M] \times\left[-C_{1}, C_{1}\right]$. Из лемм 1.4 и 1.6 следуют оценки $\left|u_{x_{2}}\right| \leqslant C_{2},\left|u_{x_{3}}\right| \leqslant C_{3}$.

Если в уравнении (1.20) функции $f_{1} \in C^{\alpha}(\Omega \times \mathbb{R} \times \mathbb{R})$ и $f_{2} \in C^{\alpha}\left(\Omega \times \mathbb{R} \times \mathbb{R}^{3}\right)$, то согласно теореме 1.1 существует решение задачи $(1.20),(1.2)$, принадлежащее $C^{2, \alpha}(\bar{\Omega})$.

2. Двумерный случай. Рассмотрим следующую задачу Дирихле:

$$
\begin{aligned}
& a(x, y, u, \nabla u) u_{x x}+2 b(x, y, u, \nabla u) u_{x y}+c(x, y, u, \nabla u) u_{y y}=f(x, y, u, \nabla u) \quad \text { в } \quad \Omega \subset \mathbb{R}^{2}, \\
& u=0 \quad \text { на } \partial \Omega,
\end{aligned}
$$

где $\nabla u=\left(u_{x}, u_{y}\right)$. Функции $a, b, c, f$ определены на множестве $\bar{\Omega} \times \mathbb{R} \times \mathbb{R}^{2}$ и принимают конечные значения при $(x, y) \in \Omega$ и конечных $u, \nabla u$. Предположим, что

$$
a \geqslant a_{0}>0, \quad c \geqslant c_{0}>0, \quad b^{2}-a c<0 \quad \text { в } \bar{\Omega} \times \mathbb{R} \times \mathbb{R}^{2},
$$

где $a_{0}$ и $c_{0}$ - некоторые постоянные. Будем считать, что $f$ представима в виде $(1.3)$, где

$$
\left|f_{1}(x, y, u, \mathbf{p})\right|<a(x, y, u, \mathbf{p}) \psi\left(\left|p_{1}\right|\right)
$$

при $(x, y) \in \Omega,|u| \leqslant M$ и конечных $\mathbf{p}=\left(p_{1}, p_{2}\right)$. Функция $\psi$ определена в п. 1 (см. (1.5)).

Лемма 2.1. Пусть и $(x, y)$ - классическое решение задачи (2.1), (2.2) такое, что $|\nabla u|<+\infty$ в $\bar{\Omega}$ и выполнены условия (2.3), (2.4), (1.6). Предположсим, что

$$
b\left(x, y, u, \nabla h_{k}\left(\zeta_{k}\right)\right) h_{k x_{i} x_{j}}\left(\zeta_{k}\right) \leqslant 0, \quad k=1,2, \quad b(x, y, u, \mathbf{p})=b(x, y, u,-\mathbf{p}) .
$$

Тогда

$$
|u(x, y)| \leqslant h_{k}\left(\zeta_{k}\right) \quad \forall(x, y) \in \bar{D}_{k}, \quad k=1,2
$$

$2 \partial e$

$$
\begin{aligned}
& \left.D_{1}=\left\{(x, y): F_{1}(y)<x<F_{1}(y)+\tau_{0},-l_{2}<y<l_{2}\right)\right\} \cap \Omega \\
& D_{2}=\left\{(x, y): G_{2}(y)-\tau_{0}<x<G_{1}(y),-l_{2}<y<l_{2}\right\} \cap \Omega .
\end{aligned}
$$

Доказательство этой леммы аналогично доказательству леммы 1.1. Функции $h_{k}, \zeta_{k}$ определены в п. 2.

Получим теперь глобальную оценку $\left|u_{x}\right|$. Предположим, что

$$
\begin{array}{r}
f_{3}\left(x, y, u, p_{1}, p_{2}\right)-f_{3}\left(\zeta, y, v, p_{1}, p_{2}\right) \geqslant 0, \\
f_{3}\left(\zeta, y, u,-p_{1}, p_{2}\right)-f_{3}\left(x, y, v,-p_{1}, p_{2}\right) \geqslant 0
\end{array}
$$

при $x \geqslant \zeta, u \geqslant v, p_{1} \geqslant 0$ и любом $p_{2}, f_{3}=f_{2} / c$. 
ЛЕмма 2.2. Пусть выполнены условия леммы 2.1, условие (2.2) и

$$
2 b^{2}-a c<0 \quad \text { в } \bar{\Omega} \times \mathbb{R} \times \mathbb{R}^{1} .
$$

Тогда для $и(x, y)$ имеем

$$
\sup _{\bar{\Omega}}\left|u_{x}\right| \leqslant C_{4}
$$

где постоянная $C_{4}$ зависит лишь от $\psi$ и $M$.

ДокАЗАТЕЛьство. Перепишем (2.1) следующим образом:

$$
\frac{a^{(x)}}{c^{(x)}} u_{x x}+2 \frac{b^{(x)}}{c^{(x)}} u_{x y}+u_{y y}=\frac{f^{(x)}}{c^{(x)}}
$$

где $g^{(z)} \equiv g(z, y, u(z, y), \nabla u(z, y))$. Рассмотрим это уравнение в точке $(x, \zeta) \in \Omega, \zeta \neq x$ :

$$
\frac{a^{(\zeta)}}{c^{(\zeta)}} u_{\zeta \zeta}+2 \frac{b^{(\zeta)}}{c^{(\zeta)}} u_{\zeta y}+u_{y y}=\frac{f^{(\zeta)}}{c^{(\zeta)}}
$$

Вычитая это уравнение из уравнения для $u(x, y)$, для функции $v(x, y, \zeta) \equiv u(x, y)-u(\zeta, y)$ получаем

$$
L^{*} v \equiv \frac{a^{(x)}}{c^{(x)}} v_{x x}+2 \frac{b^{(x)}}{c^{(x)}} v_{x y}+\frac{a^{(\zeta)}}{c^{(\zeta)}} v_{\zeta \zeta}+2 \frac{b^{(\zeta)}}{c^{(\zeta)}} v_{\zeta y}+v_{y y}=\frac{f^{(x)}}{c^{(x)}}-\frac{f^{(\zeta)}}{c^{(\zeta)}}
$$

Условие (2.7) обеспечивает эллиптичность оператора $L^{*}$. Действительно,

$\operatorname{det}\left(\begin{array}{ccc}\frac{a^{(x)}}{c^{(x)}} & \frac{b^{(x)}}{c^{(x)}} & 0 \\ \frac{b^{(x)}}{c^{(x)}} & 1 & \frac{b^{(\zeta)}}{c^{(\zeta)}} \\ 0 & \frac{b^{(\zeta)}}{c^{(\zeta)}} & \frac{a^{(\zeta)}}{c^{(\zeta)}}\end{array}\right)=\frac{1}{2}\left(\frac{a^{(x)}}{c^{(x)}}\left[\frac{a^{(\zeta)}}{c^{(\zeta)}}-2\left(\frac{b^{(\zeta)}}{c^{(\zeta)}}\right)^{2}\right]+\frac{a^{(\zeta)}}{c^{(\zeta)}}\left[\frac{a^{(x)}}{c^{(x)}}-2\left(\frac{b^{(x)}}{c^{(x)}}\right)^{2}\right]\right)>0$.

Аналогично лемме 1.2 завершаем доказательство.

Предположим, что при $(x, y) \in \Omega,|u| \leqslant M,\left|p_{1}\right| \leqslant C_{4}$ и конечном $p_{2}$ выполняется

$$
\left|f_{1}\left(x, y, u, p_{1}, p_{2}\right)\right|<a\left(x, y, u, p_{1}, p_{2}\right) \psi\left(\left|p_{2}\right|\right) .
$$

ЛЕмма 2.3. Пусть $и(x, y)$ - классическое решение задачи (2.1), (2.2) такое, что $|\nabla u|<+\infty$ в $\bar{\Omega}$. Предположим, что условия (2.3)-(2.8) выполнены. Тогда

$$
|u(x, y)| \leqslant h_{k}\left(\zeta_{k}\right) \quad \forall(x, y) \in \bar{D}_{k}, \quad k=3,4,
$$

$2 \partial e$

$$
\begin{aligned}
& D_{3}=\left\{(x, y): F_{2}(x)<y<F_{2}(x)+\tau_{0},-l_{1}<x<l_{1}\right\} \cap \Omega, \\
& \left.D_{4}=\left\{(x, y): G_{2}-\tau_{0}<y<G_{2},-l_{1}<x<l_{1}\right)\right\} \cap \Omega .
\end{aligned}
$$

Доказательство этой леммы аналогично доказательству леммы 1.1.

Сформулируем условия, гарантирующие глобальную оценку $\left|u_{y}\right|$. Предположим, что

$$
\begin{aligned}
f_{4}\left(x, y, u, p_{1}, p_{2}\right)-f_{4}\left(x, \zeta, v, p_{1}, p_{2}\right) & \geqslant 0 \\
f_{4}\left(x, \zeta, u, p_{1},-p_{2}\right)-f_{4}\left(x, y, v, p_{1},-p_{2}\right) & \geqslant 0
\end{aligned}
$$

при $y \geqslant \zeta, u \geqslant v, p_{2} \geqslant 0$ и любом $p_{1}, f_{4}=f_{2} / a$. 
ЛЕмма 2.4. Пусть выполнены условия леммы 2.3 и условие (2.9). Тогда для $u(x, y)$ имеет место оценка

$$
\sup _{\bar{\Omega}}\left|u_{y}\right| \leqslant C_{5}
$$

где постоянная $C_{5}$ зависит лишь от $\psi$ и $M$.

Доказательство леммы 2.4 аналогично доказательству леммы 2.1.

Сформулируем теорему существования.

ТЕорема 2.1. Предположим, что $a, b, c, f \in C^{\alpha}\left(\Omega \times \mathbb{R} \times \mathbb{R}^{2}\right)$ для некоторого $\alpha \in(0,1)$ и выполнены условия (2.3)-(2.9). Предположим также, что выполнено условие, гарантирующее оценку $|u| \leqslant M$. Тогда в любой строго выпуклой области $\Omega$ задача (2.1), (2.2) имеет по крайней мере одно решение $u(x, y) \in C^{2, \alpha}(\bar{\Omega})$. Если функиия $f$ возрастает по переменной $u$, а функиии $a, b, c$ не зависят от $u$, то такое решение единственно.

ДокАЗАТЕЛЬСтво. В силу полученных оценок и условия (2.3) уравнение (2.1) можно рассматривать как линейное строго эллиптическое уравнение

$$
\tilde{a}(x, y) u_{x x}+2 \tilde{b}(x, y) u_{x y}+\tilde{c}(x, y) u_{y y}=\tilde{f}(x, y)
$$

с ограниченньми коэффициентами $\tilde{a}, \tilde{b}, \tilde{c}$ и правой частью $\tilde{f}$. Из теоремы 11.4 [2] следует, что решение задачи Дирихле для этого уравнения (при гладких краевых условиях и гладкой границе) удовлетворяет неравенству

$$
|u|_{C^{1+\delta}(\bar{\Omega})} \leqslant C\left(\sup _{\Omega}|u|+\sup _{\Omega}\left(\frac{|\tilde{f}|}{\lambda}\right)\right), \quad \delta \in(0,1),
$$

где $\lambda$ - минимальное собственное значение матрицы старших коэффициентов. Постоянная $C$ зависит лиш от верхних граней модулей коэффициентов и правой части. Из этой оценки и теоремы 13.8 [2] следует сушествование требуемого решения.

В заключение приведем уравнение, на которое могут быть распространены результаты п. 1. Рассмотрим задачу Дирихле

$$
a_{i j}\left(x_{i}, x_{j}, \nabla u\right) u_{x_{i} x_{j}}=f(\mathbf{x}, u, \nabla u) \text { в } \Omega \subset \mathbb{R}^{3}, \quad u=0 \text { на } \partial \Omega ;
$$

здесь $a_{i i}=a_{i i}\left(x_{i}, \nabla u\right)$. Предполагаем, что для $\mathbf{x} \in D_{k}, k=1,2,|u| \leqslant M$ и произвольных $\mathbf{p}$ вьполняется

$$
\begin{aligned}
& \sum_{i, j=1, i \neq j}^{3} a_{i j}\left(x_{i}, x_{j}, \nabla h_{k}\left(\zeta_{k}\right)\right) h_{k x_{i} x_{j}}\left(\zeta_{k}\right) \leqslant 0, \\
& a_{i j}\left(x_{i}, x_{j}, \mathbf{p}\right)=a_{i j}\left(x_{i}, x_{j},-\mathbf{p}\right) \quad \text { для } i \neq j,
\end{aligned}
$$

это многомерный аналог условия (2.5). Кроме того, необходимо требовать выполнение многомерного аналога условия (2.7). Это условие, сформулированное в [12], очень громоздко, и мы не будем приводить его здесь; отметим лишь, что оно выполнено если $a_{i j} \equiv 0$ при $i \neq j$. Полагаем, что $a_{i j} \in C^{1}\left(\Omega \times \mathbb{R}^{3}\right), f \in C^{\alpha}\left(\Omega \times \mathbb{R} \times \mathbb{R}^{3}\right)$. Трудности, возникающие за счет появления коэффищиентов $a_{i j}\left(x_{i}, x_{j}, \nabla u\right)$, могут быть преодолены точно так же, как и в [12].

Отметим, что специфика двумерного случая, как видно из теоремы 2.1 , позволяет рассматривать уравнения с коэффищиентами, зависящими от всех переменных. Кроме того, требование дифференцируемости старших коэффициентов можно заменить на условие их непрерьвности по Гёльдеру. 


\section{СПИСОК ЦИТИРОВАННОЙ ЛИТЕРАТУРЫ}

[1] Ладыженская О. А., Уральцева Н. Н. Линейные и квазилинейные уравнения эллиптического типа. М.: Наука, 1973.

[2] Гилбарг Д., Трудингер Н. С. Эллиптические дифференциальные уравнения с частными производными второго порядка. М.: Наука, 1989.

[3] Крылов Н. В. Нелинейные эллиптические и параболические уравнения второго порядка. M.: Наука, 1985.

[4] Бернштейн С. Н. Об уравнениях вариационного исчисления. Собр. сочинений. Т. 3. М.: Изд-во АН СССР, 1960.

[5] Amman H., Crandall M. G. On some existence theorems for semi-linear elliptic equations // Indiana Univ. Math. J. 1978. V. 27. № 5. P. 779-790.

[6] Похожаев С. И. Об уравнении вида $\Delta u=f(\mathbf{x}, u, \nabla u) / /$ Матем. сб. 1980. Т. $113(155)$. № $1(10)$. C. $324-338$.

[7] Tersenov Al.S. Estimate of the solution of the Dirichlet problem for parabolic equations and applications // J. Math. Anal. Appl. 2002. V. 273. № 1. P. 206-216.

[8] Кружков С. Н. Квазилинейные параболические уравнения и системы с двумя независимыми переменньми // Тр. семин. им. И. Г. Петровского. 1979. № 5. С. 217-271.

[9] Камынин В. Л. Априорные оценки и глобальная разрешимость квазилинейных параболических уравнений // Вестн. МГУ. Сер. 1. Матем., мех. 1981. №1. С. 33-38.

[10] Камынин В. Л.Априорные оценки решений квазилинейных параболических уравнений на плоскости и их приложения // Дифференц. уравнения. 1983. Т. 19. № 5. С. 590-598.

[11] Хуснутдинова Н. В. Об условиях ограниченности градиента решения вырождающихся параболических уравнений // Динамика сплошной среды. 1985. Т. 71. С. 120-129.

[12] Tersenov Al.S. On quasilinear non-uniformly elliptic equations in some non-convex domains // Comm. Partial Differential Equations. 1998. V. 23. № 11. P. 2165-2186.

[13] Tersenov Al.S. On quasilinear non-uniformly parabolic equations in general form // J. Differential Equations. 1998. V. 142. № 1. P. 263-276.

[14] Tersenov Al.S., Tersenov Ar. S. The Cauchy problem for a class of quasilinear parabolic equations // Ann. Mat. Pura Appl. 2003. V. 182. № 3. P. 325-336.

Университет о. Крит, Греция 